\title{
Método para el gobierno de las tecnologías de la información basado en la modelización empresarial
}

\author{
Method for it governance based on enterprise modeling
}

\author{
Eloy Hontoria ', Antonio Fernández², Ma Victoria de la Fuente' y Lorenzo Ros ' \\ I Grupo de Investigación «Gestión e Ingeniería de Organización». ETSII-Universidad Politécnica de Cartagena \\ C/Dr. Fleming s/n, 30202 Cartagena. eloy.hontoria@upct.es \\ 2 Área Ciencias de la Computación e Inteligencia Artificial. Universidad de Almería
}

Fecha de recepción: 7-9-2011

Fecha de aceptación: 19-9-2011

Resumen: En el actual entorno de continua evolución de las tecnologías de la información y las comunicaciones (TIC)*, y como respuesta a los conceptos referentes al Gobierno de las Tecnologías de la Información (TI), en el presente trabajo se define y diseña un método que posibilite el cambio en una organización hacia los nuevos paradigmas de diseño y gestión de las TI, y sobre todo en su interacción con las redes interorganizacionales. La aplicación del método definido sobre la organización del servicio de las tecnologías de la información de una Universidad ha posibilitado la integración de distintas herramientas (por ejemplo modelo GTI4U, Modelo de Madurez, Metodología de Modelización Empresarial) en el proceso de gobierno de las TI, merced a la modelización de los procesos de negocio y los flujos relacionados con ello.

Palabras clave: modelización empresarial, método para el gobierno de las TI.

\begin{abstract}
Nowadays, the evolving environment and continuous evolution of Information and Communication Technologies (ICT)* and as a response to the concepts relating to IT governance, this paper defines and designs a method that facilitates the change in an organization towards the new paradigms of IT design and management, and deals mainly in their interaction with inter-organization networks. The application of the defined method on the organization of the information and communications service of a University has facilitated the integration of different tools (for example GTI4U model, Maturity Model, Enterprise Integration Methodology) in the IT governance process, thanks to the modelization of the business processes and the flows related with this.
\end{abstract}

Key Words: enterprise modelling, method for IT governance.

\section{Introducción}

Las Tecnologías de la Información (TI) fueron cuestionadas en su momento por falta de productividad (Roach, 1987), o por su falta de importancia en la organización (Carr, 2003). En la actualidad se considera que las TI aportan valor a las organizaciones hasta el punto de proporcionar ventaja competitiva (Porter 2006). Las TI únicamente incrementan el rendimiento de aquellas organizaciones que son capaces de gobernarlas de forma adecuada (Weill y Ross, 2004).

Según Benemati et al. (1997), las TI evolucionan de forma rápida, siendo cada vez más importante el im- pacto estratégico de las TI sobre la operativa de las organizaciones, así como la gestión adecuada de las mismas. Olugbode et al. (2007) propusieron tipificar el rol de las TI en una organización como el ajuste o alineamiento con los objetivos estratégicos de la organización. Las TI únicamente pueden estar bien alineadas si la infraestructura que se ha utilizado para implementar la estrategia de TI es la adecuada. Por ello, la estrategia de las TI debe dar soporte tanto a la estrategia de la organización como a los procesos de negocio de la misma.

La gestión y dirección global de una organización está basada en el gobierno corporativo y en el gobierno

\footnotetext{
* La norma ISO 38500, en el punto I.6.7 expone: «... el término TI incluye las Tecnologías de la Comunicación (TC) y el término compuesto «Tecnologías de la Información y las Comunicaciones»».
} 
de sus TI (Hamaker, 2003). Ambos gobiernos se basan en los mismos principios (Hamaker y Hutton, 2004; De Haes y Van Grembergen, 2005).

El gobierno de las TI es la capacidad que tiene la organización, cuya responsabilidad recae en el Equipo de Gobierno, para dirigir el diseño y la implementación de las TI; asegurando de esta forma su alineamiento con la estrategia de negocio (Van Grembergen, 2004). El gobierno de las TI propone incorporar las decisiones que conciernen a $\mathrm{TI}$ a la agenda de los Consejos de Dirección para poder dirigirlas y conseguir de esa forma la alineación de las estrategias TI con las estrategias de negocio.

Para responder a las influencias de su entorno, la organización necesita recurrir a modelos de organización y gestión que le proporcionen la agilidad suficiente para elaborar respuestas inmediatas mediante la explotación eficiente y efectiva de sus recursos de TI.

La organización obtiene un valor de retorno en forma de ahorro de costes, mejorando su organización interna, satisfacción de los usuarios, imagen corporativa y la proyección externa de la organización. El principal beneficio que obtiene es un incremento en la agilidad para afrontar los cambios que debe abordar la organización (De Haes y Van Grembergen, 2005).

\section{Paradigmas de gobierno de las TI}

El concepto «Gobierno de las Tl» nace a mediados de los 90 y ha ido madurando desde entonces, abarcando cada vez más temas y áreas, hasta convertirse en una disciplina en sí misma. Este término fue definido inicialmente por Loh y Venkatraman (1992), y posteriormente por Henderson y (1993), para describir al conjunto de mecanismos que aseguran lograr las capacidades de las TI necesarias para la óptima operación de los procesos de negocio.

En una organización, el buen gobierno corporativo pasa por la gestión y dirección de sus tecnologías de la información. Los directivos de una organización deben comprender la oportunidad de convertir sus $\mathrm{Tl}$ en elementos estratégicos alineados con el resto de la organización.

Según Van Grembergen, el gobierno de la TI es la capacidad que tiene la organización para dirigir el diseño y la implementación de la estrategia de las TI, asegurando que se alinea con la estrategia del ne- gocio (Van Grembergen, 2004). La responsabilidad del gobierno de las TI recae tanto en el Equipo de Gobierno como en los directores de las TI.

Las Universidades Españolas, al igual que cualquier otra organización, necesitan implantar sistemas de gobierno de las $\mathrm{Tl}$, al objeto de mejorar el rendimiento de la organización. Entre las iniciativas recientes de la Conferencia de Rectores (CRUE) está promover el desarrollo de sistemas de gobierno de las TI en las Universidades Españolas (Uceda, 2008). En este caso específico, los pasos para implementar el gobierno de las TI en una organización fueron explicitados porVan Grembergen y De Haes (2008):

- Formación de los máximos directivos en gobierno de las TI dentro de la organización.

- Análisis y conocimiento de la «madurez» de la situación inicial del gobierno de las TI.

- Diseño de un plan de implantación del gobierno de las TI en la organización.

Posteriormente Fernández (20 I0), propone un modelo a partir del marco de referencia definido por Van Grembergen y De Haes. Este modelo de gobierno de TI para Universidades (GTI4U) está basado en la norma internacional ISO/IEC 38500:2008, que define el gobierno de las TI como «sistema mediante el que se dirige y controla la utilización de la TI actuales y futuras» (ISO 38500, 2008). Supone la dirección y evaluación de los planes de utilización de las TI que dan soporte a la organización y monitorización de dicho uso para alcanzar lo establecido en los planes. Establece los principios de un buen gobierno de las TI desde la visión de la Alta Dirección.

El modelo GTI4U se centra en el segundo paso, especificado por Van Grembergen y De Haes (2008) de análisis y conocimiento de la «madurez» de la situación del gobierno de las TI. Dicho modelo incorpora un proceso de análisis muy detallado que evalúa los distintos aspectos de las TI en la organización, así como utiliza el concepto de modelo de madurez que incorpora la norma ISO 38500.

\section{Gobierno de las TI en la Universidad Española}

El objetivo fundamental de la investigación que se presenta en este trabajo es mejorar el gobierno, la administración y la planificación de las TI en las Universidades Españolas mediante el desarrollo de un 
método. Dicho método integra los distintos modelos que se han desarrollado, basándose en una metodología de Modelización Empresarial, y llegando a las siguientes conclusiones:

- La existencia de modelos de análisis, planificación y gobierno de las TI en Universidades españolas e internacionales es muy escasa, no conformando una base de conocimiento amplia.

- La situación actual de las Tecnologías de la Información, la cultura de la planificación estratégica y las iniciativas en procesos de calidad en la Universidad Española requieren nuevas herramientas que ayuden a organizaciones complejas a establecer procedimientos que les permita implantar progresivamente sistemas de gobierno de las TI.

- La utilización del método diseñado, que contribuye al proceso de alineación de la TI y los objetivos de la organización, así como el análisis de valor que proporcionan las $\mathrm{TI}$ a las organizaciones.

\section{Metodología de Modelización Empresarial}

Para el desarrollo del método, se utilizó la metodología de Modelización Empresarial ERE-GIO (de la Fuente et al., 2008; de la Fuente et al., 20 I 0), metodología que permite el redescubrimiento de cualquier entidad de negocio estableciendo dos fases generales de trabajo (fases de ingeniería inversa y de ingeniería directa), incluyendo una serie de etapas que permiten el estudio tanto de la situación actual de la organización como el plan posterior de evolución hacia un nuevo modelo o situación de las TI.

La metodología de Modelización Empresarial EREGIO se basa en la teoría de los ciclos de vida aplicados a las entidades de negocio. Dicha metodología ha sido diseñada para ser utilizada como herramienta que permite caracterizar y rediseñar la organización a partir del valor de los elementos que la componen: recursos, procesos, tecnología, información, etc. Así mismo, la metodología ERE-GIO enfoca la modeli-

Fases y etapas de la Metodología ERE-GIO

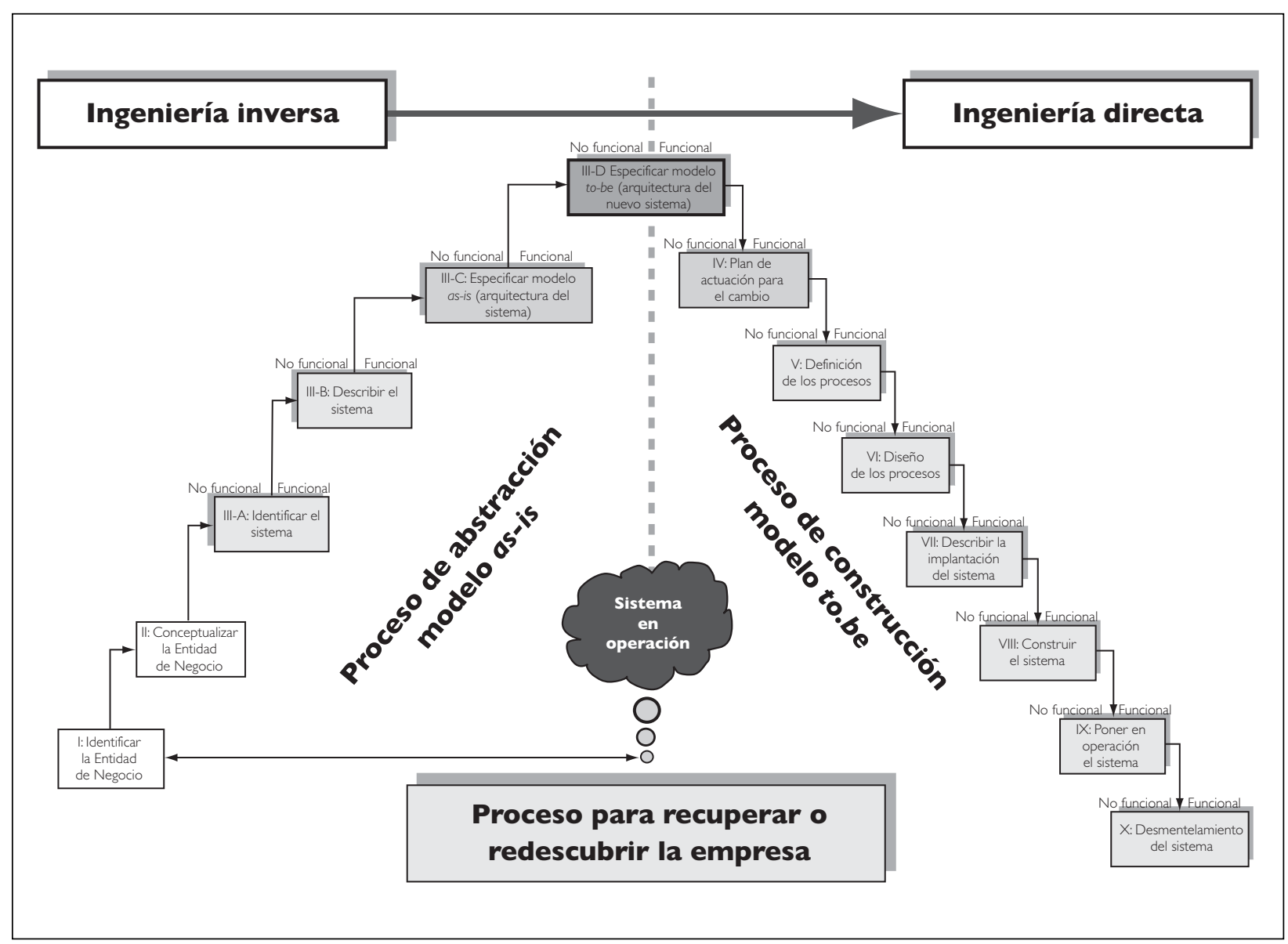


zación empresarial de forma que se puede aplicar a un proceso de evolución de la organización, mediante el seguimiento del ciclo de vida para el desarrollo de nuevos sistemas.

Esta metodología permite definir el modelo «as-is», utilizando para ello el modelo de madurez GTI4U para el gobierno de las TI. La construcción del modelo «as-is» se desarrolla siguiendo la fase de Ingeniería Inversa, que consigue identificar y medir las variables que definen la situación actual del sistema, permitiendo su modelado.

Para proponer el modelo «to-be», o como se quiere que funcione el sistema, se incorporarán los aspectos que los directivos de las TI de la organización desean incorporar al gobierno de las TI. Este modelo «to-be», siguiendo la fase de Ingeniería Directa de la metodología, permite mediante la desagregación de la información realizada en la fase inversa, establecer un plan de implantación para este nuevo modelo.

\section{I. Fase de Ingeniería Inversa. Modelo GTI4U}

En esta fase se delimita el marco estratégico de la entidad de negocio, en este caso concreto el gobierno de las TI, facilitando el enfoque de procesos, buscando la satisfacción del cliente (externo o interno de la entidad) y eliminando aquellas actividades que no contribuyan a dicho objetivo. Esta fase se centra en definir la secuencia de actividades que permiten enfocar adecuadamente los procesos de mejora.

En la fase de ingeniería inversa se analizan los procesos que realiza la entidad de negocio seleccionada, para desarrollar el modelo «as-is» de dicho sistema. Este modelo «as-is» se conceptualiza en base al modelo de madurez de $\mathrm{TI}$, con objeto de realizar la propuesta de modelo «to-be».

Para ello se parte del modelo GTI4U diseñado y validado por Fernández (2010). Este modelo se basa en la ISO 38500, cuyos principios son: Responsabilidad, Estrategia, Adquisición, Desempeño, Cumplimiento, y Factor Humano.

Estos principios se desarrollan en los modelos de madurez del modelo GTI4U, que soporta el proceso de abstracción y que permite la construcción del modelo «as-is» de la organización.

Las etapas de la fase de ingeniería inversa, que se han de seguir con el soporte del modelo GTI4U, son: i. Identificación de la entidad de negocio. Selecciona la parte de la organización o entidad de negocio en que se desarrollará el análisis.

ii. Conceptualización de la entidad de negocio. En esta etapa se establece el marco estratégico en el cual queda definida la entidad de negocio seleccionada y que permite establecer las bases para el posterior desarrollo del modelo «to-be».

iii. Análisis de los procesos. Esta etapa realiza una descripción de los procesos de negocio, centrándose en los que se están desarrollando actualmente (modelo «as-is») y sus características más relevantes.

Como se puede ver, la etapa 3 es la etapa fundamental de esta fase de Ingeniería Inversa, ya que especifica los procesos actuales (modelo «as-is»), así como los deseados (modelo «to-be») de la entidad de negocio. No se debe confundir con la definición y diseño de la nueva forma de operar de la entidad de negocio, cuya implantación es el objeto principal de la fase de ingeniería directa.

\subsection{Fase de Ingeniería Directa. Modelo PTI4U}

La segunda fase de la metodología, denominada de ingeniería directa, se encarga de la ejecución planificada del desarrollo, implantación y puesta en marcha de los procesos (redefinidos y/o nuevos) del nuevo modelo de funcionamiento (modelo «to-be») de la entidad de negocio. El modelo PTI4U utiliza las guías propuestas por la ISO 38500 como base para diseñar su propio catálogo de buenas prácticas.

Esta fase de ingeniería directa está compuesta por seis etapas, que son:

iv. Plan de actuación para el cambio. En esta etapa se establece el plan de actuación para abordar la transición entre un modelo y el otro. El objeto principal de esta etapa es establecer el marco y las condiciones que definen el comportamiento del nuevo sistema y señalar como se realizará la gestión del cambio. En base a los siguientes pasos:

v. Definición de los procesos. Consiste en el establecimiento, por parte del usuario y/o responsable de cada proceso, de las características y actividades a desarrollar en ellos.

vi. Diseño de los procesos. Consiste en diseñar todas las actividades que permitirán que se cum- 
plan los requerimientos definidos por el usuario en los procesos de la etapa anterior, mediante los siguientes pasos:

vii. Descripción de la implementación del sistema. Se realiza una selección de las técnicas, herramientas y tecnologías más adecuadas que proporcionen los componentes a utilizar en una realización integrada y efectiva del sistema.

viii. Construcción del sistema. Seleccionados los elementos adecuados para llevar a cabos los requisitos, se pasa al desarrollo e instalación de estos en la entidad seleccionada.

ix. Puesta en operación del sistema. Comprende la ejecución de todas las actividades de la entidad, especificadas en las etapas anteriores, con los recursos de la etapa anterior con el fin de conseguir los objetivos deseados.

Figura 2

Método para el gobierno de las TI basado en la Modelización Empresarial

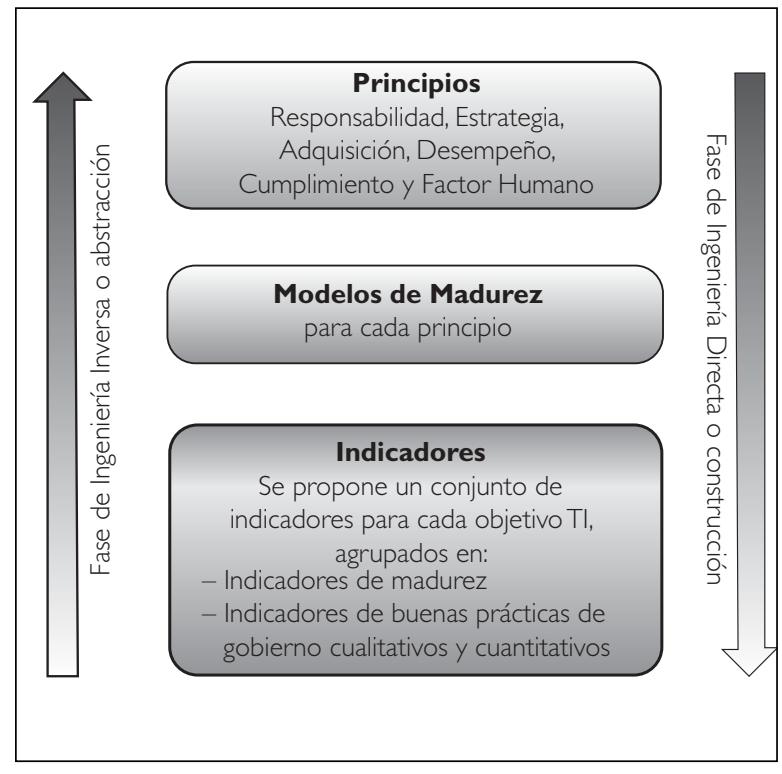

\section{Conclusiones}

La organización de las TI está en constante evolución y adaptación a los requerimientos de los mercados, para controlar ese proceso de cambio son necesarias herramientas y técnicas, y de forma más especifica métodos que permitan el análisis de los sistemas actuales y que ayuden al diseño y construcción de nuevos funcionamientos de las Tecnologías de la Información.
Existen diferentes métodos para la modelización de los procesos de negocio, según se quiera representar diferentes escenarios o vistas de la organización. En este trabajo se ha utilizado la metodología EREGIO, para su aplicación al proceso de modelización de los procesos de gobierno de las TI.

La metodología ERE-GIO, debido a la definición de las dos fases de Ingeniería condiciona su aplicación en el análisis tanto del proceso actual de gobierno como del que se desea introducir.

El modelo GTI4U ofrece un procedimiento de abstracción que da soporte a la construcción del modelo «as-is» de gobierno de las TI en la organización considerada.

El modelo GTI4U junto con las guías de la norma ISO 38500, han permitido construir una base de buenas prácticas, que a su vez permiten el modelo directo y la construcción del nuevo sistema de gobierno de las TI (modelo PTI4U).

\section{Bibliografía}

BENEMATI, J., LEDERER, A, SINGH, M. (1997), Changing information technology. Information Technology Management, 3I (5): 276-288.

CARR, N .G. (2003), It doesn't matter. Harvard Business Review 8I (5): May 2003.

DE HAES, S., VAN GREMBERGEN, W. (2005), IT governance structures, processes and relational mechanisms - achieving IT - Business alignment in a major Belgian financial group. Proceedings of the $38^{\mathrm{a}}$ Hawaii International Conference on System Science. IEEE. C.S.

DE LA FUENTE, M.V., ROS, L., CARDÓS, M. (2008), Integrating forward and reverse supply chains: Application to a metal-mechanic company. International Journal of Production Economics, I I I, pp.782-792.

DE LA FUENTE, M.V., ROS, L., ORTIZ, A. (20I0), Enterprise modelling methodology for forward and reverse supply chain flows integration. Computers in Industry, 61, pp 702-710.

FERNÁNDEZ, A. (20|0), Gobierno de las TI para Universidades. CRUE.

HAMAKER, S. (2003), Spotlight on governance. Information System Control Journal, Volume I.

HAMAKER, S., HUTTON, A. (2004), Principles of IT governance. Information System Control Journal,Volume 2.

HENDERSON, J.C., VENKATRAMAN, N. (1993), Strategic alignment: leveraging information technology for 
transforming organisations. IBM Systems Journal, 32(I), pp 4-16.

ISO 38500 (2008), ISO/IEC 38500:2008 Corporate Governance of Information Technology. ISO/IEC. 2008, from: www.iso.org.

LOH, L., VENKATRAMAN, N. ( 1992), Diffusion of information technology outsourcing: influence sources and the Kodak effect. Information Systems Research, 3(4), pp 334-359.

OLUGBODE, M., RICHARDS, R., BISS, T. (2007), The role of information technology in achieving the organisations strategic development's goals: a case study. Information Systems, 32.

PORTER, M. (2006), Tecnología y ventaja competitiva. Estrategia y ventaja competitiva Ed. Deusto, pp ||3-17|.
ROACH, S. ( 1987), American's technology dilemma: a profile of the information economy. Morgan Stanley Special Economics Study, April 1987. New York.

UCEDA, J. (2008), Estrategia de la CRUE en relación a la implantación de modelos de gobierno de las TI en las Universidades Españolas. Seminario gobierno de las TI en las Universidades Españolas. Sectorial TIC de la CRUE. Universidad Politécnica de Madrid.

VAN GREMBERGEN, W. (2004), Strategies for information technology governance. Idea group.

VAN GREMBERGEN,W., DE HAES, S. (2008), Implementing Information Technology Governance. Models, Practices and Cases. IGI Publishing.

WEILL, P., ROSS, J.W. (2004), IT Governance: How Top Performers Manage IT Decision Rights for Superior Results. Harvard Business School Press. 